\title{
The nearby QSO host I Zw 1: the stellar disk and adjacent objects ${ }^{\star}$
}

\author{
J. Scharwächter ${ }^{1}$, A. Eckart ${ }^{2}$, S. Pfalzner ${ }^{2}$, I. Saviane ${ }^{1}$, and J. Zuther ${ }^{2}$ \\ 1 European Southern Observatory, Casilla 19001, Santiago 19, Chile \\ e-mail: jscharwa@eso.org \\ 2 I. Physikalisches Institut, Universität zu Köln, Zülpicher Str. 77, 50937 Köln, Germany \\ Received 7 September 2006 / Accepted 9 May 2007
}

\begin{abstract}
Context. The relation between tidal interactions, starbursts, and the onset and/or fueling of active galactic nuclei (AGN) is a matter of debate. I Zw 1 is considered as the prototypical narrow-line Seyfert 1 galaxy (NLS1) and as one of the closest quasi-stellar objects (QSOs). With a clear spiral host and a small companion galaxy to the west, I Zw 1 is a possible example of minor-merger-related nuclear activity.

Aims. This study investigates possible signs of a relation between merger process, star formation activity, and AGN properties in the case of I Zw 1.

Methods. The morphology of I Zw 1 and nearby sources is investigated via high-resolution near-infrared (NIR) images. Color trends in the host galaxy of I Zw 1 are discussed by means of optical-to-NIR color composites. Long-slit spectra of the QSO nucleus of I Zw 1 and of the two nearby sources to the north and the west of the I Zw 1 disk are analyzed.

Results. The data support the scenario of a tidal interaction between I Zw 1 and the small companion galaxy to the west. A concentration of blue color in the western part of the I Zw 1 host galaxy might be the manifestation of merger-induced star formation activity. Previous findings that the likely companion has an old evolved stellar population are substantiated by the new data. An extension to the west of the putative companion emerges as a separate source. The source to the north of the I Zw 1 disk is reconfirmed as a late-type foreground star. Lines in the nuclear $K$-band spectrum of I Zw 1 are discussed in comparison to data prior to this article and line fluxes are reported.
\end{abstract}

Key words. galaxies: quasars: individual: I Zw 1 - galaxies: Seyfert - galaxies: interactions - methods: observational

\section{Introduction}

It is widely accepted that nuclear activity in galaxies is caused by mass accretion onto a supermassive black hole (see e.g. review by Urry 2004). Furthermore, it is known that AGN are often connected to star formation activity (e.g. Kauffmann et al. 2003). However, the importance of tidal interactions for the onset and/or fueling of AGN and starbursts is still unclear (e.g. Dunlop et al. 2003; Grogin et al. 2005; Koulouridis et al. 2006).

Regarding the comparable bolometric luminosities and local space densities of ULIRGs and local QSOs, Sanders et al. (1988) proposed an evolutionary link between the two classes of objects. In this scenario, galaxy mergers are assumed to cause the gas inflow which triggers an intense starburst and a dustenshrouded AGN. At this stage, the object would appear as ULIRG. When winds and feedback blow away the dust from the central region, the object goes through an optically bright QSOphase. Such an evolutionary scenario is supported by recent numerical simulations which includesupermassive black holes and

$\star$ Based on observations collected at the Very Large Telescope (UT1) of the European Southern Observatory, Paranal (Chile), under service mode project 67.B-0009. Complementary data are based on observations made with the $3.6 \mathrm{~m}$ telescope of the European Southern Observatory, La Silla (Chile), and with the NASA/ESA Hubble Space Telescope, obtained from the ESO/ST-ECF Science Archive Facility (joint collaboration of the European Southern Observatory and the Space Telescope - European Coordinating Facility). feedback (e.g. Di Matteo et al. 2005; Hopkins et al. 2005, 2006). Observations show that virtually all ULIRGs are found in major merger systems (e.g. Bushouse et al. 2002; Veilleux et al. 2002). But on the side of QSOs, the importance of mergers is far less conclusive. In fact, there is increasing evidence that the results might depend on the selection of the QSO sample. A comparison of ULIRGs with QSOs from the radio-loud/radio-quiet sample by Dunlop et al. (2003), which are mainly located in massive elliptical host galaxies, shows that both populations occupy different parts of the fundamental plane (Tacconi et al. 2002). Palomar Green QSOs, instead, are discussed as putative candidates for a post-ULIRG stage (e.g. Surace et al. 2001; Haas et al. 2003; Dasyra et al. 2007). An exception, however, is the prominent fraction of Palomar Green QSOs with clear spiral host galaxies. According to today's consensus from numerical simulations, major mergers in the local Universe typically result in elliptical merger remnants (e.g. Hernquist 1992; Naab \& Burkert 2003; González-García \& Balcells 2005; Naab \& Trujillo 2006). Spiral hosts are very unlikely to have undergone a recent major merger.

Nuclear activity in spiral host galaxies might instead be related to minor mergers with low-mass companion galaxies. Numerical simulations show that minor mergers can cause large amplitude spirals and radial gas inflow in the primary galaxy (Hernquist \& Mihos 1995). Hernquist \& Mihos (1995) report that this gas could be available for the fueling of a nuclear starburst and AGN activity but that the gas inflow rates during the early stages of the merger are low. Consequently, central activity might only be induced in the late stages of the merger, i.e. when 


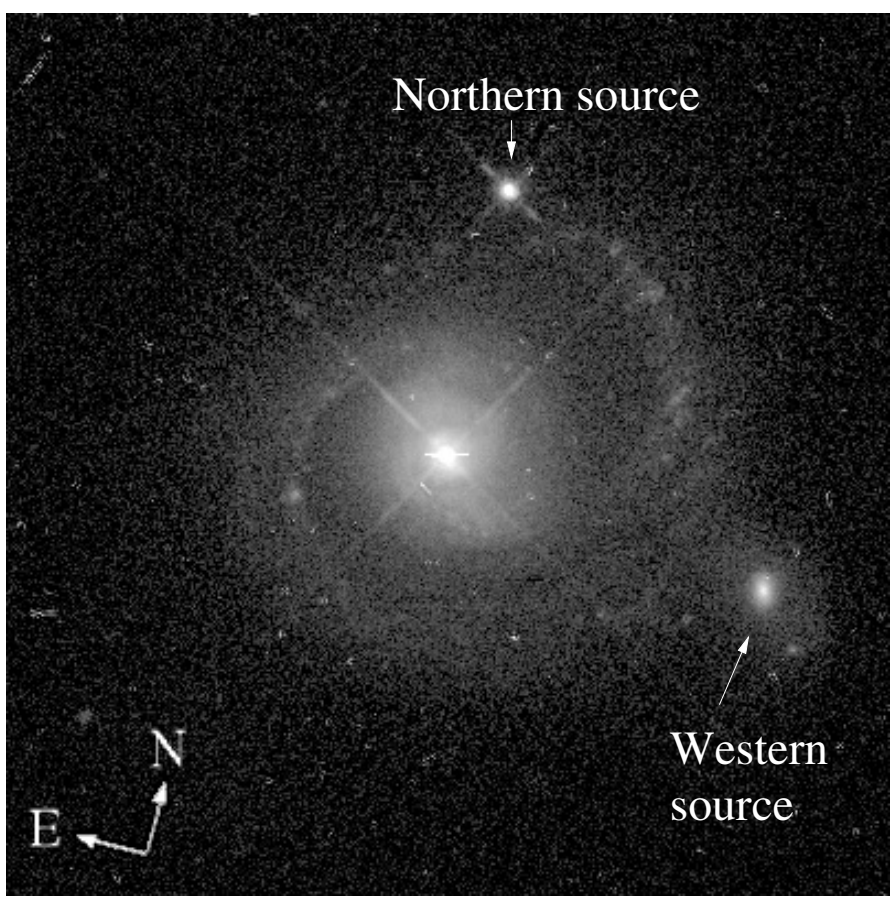

Fig. 1. HST image of I Zw 1 based on observations with the WFPC2 camera in the $F 814 W$ filter, obtained from the ESO/ST-ECF Science Archive Facility. For this plot, the image has been partially cleaned from cosmic rays and processed with an unsharp masking technique.

the satellite galaxy has reached the central few kiloparsecs of the primary galaxy. The different observational studies about a possible excess of companions around Seyfert galaxies yield extremely contradictory results (Dahari 1984; Rafanelli et al. 1995; Laurikainen \& Salo 1995; de Robertis et al. 1998; Dultzin-Hacyan et al. 1999). Rather than triggering nuclear activity, minor mergers may instead increase the luminosity of a pre-existing AGN to QSO-levels (Corbin 2000). Several observables of low-luminosity Seyfert galaxies can also be reproduced by numerical simulations of a merger-independent model (Hopkins \& Hernquist 2006). In this model, the fueling process is based on the stochastic accretion of cold gas.

In view of the above controversies, I $\mathrm{Zw} 1$ is an interesting example for a detailed observational case study. I Zw 1 is classified as a NLS1 as well as an infrared-excess Palomar Green QSO and a possible candidate for an ongoing minor merger. With $M_{B}=-22.62$ (Canalizo \& Stockton 2001), the nuclear blue magnitude of I Zw 1 just meets the QSO criterion $M_{B}<-22.1$ (Schmidt \& Green 1983) ${ }^{1}$. At a redshift of only $z=0.0611$ (Condon et al. 1985), I Zw 1 is therefore considered as one of the closest QSOs. As illustrated by the Hubble Space Telescope (HST) image in Fig. 1, the spiral host galaxy of I Zw 1 is clearly visible. The host of I Zw 1 shows direct observational evidence for young stellar populations and ongoing nuclear star formation activity (e.g. Hutchings \& Crampton 1990; Eckart et al. 1994; Schinnerer et al. 1998; Canalizo \& Stockton 2001; Scharwächter et al. 2003). The nuclear starburst can be modeled by a $4.5 \times 10^{7}$ yrs-old decaying starburst with a decay time of $5 \times 10^{6} \mathrm{yrs}$ and an upper mass cut-off of $\geq 90 \mathcal{M}_{\odot}$, which up to now would have produced $1.9 \times 10^{10} \mathcal{M}_{\odot}$ (Schinnerer et al. 1998). This nuclear starburst might be associated with a circum-nuclear molecular ring in ${ }^{12} \mathrm{CO}(1-0)$, (Schinnerer et al. 1998; Staguhn et al. 2004). Furthermore, I Zw 1 shows

\footnotetext{
${ }^{1}$ Assuming $H_{0}=75 \mathrm{~km} \mathrm{~s}^{-1} \mathrm{Mpc}^{-1}$ and $q_{0}=0.5$.
}

indications for an ongoing minor merger with the companion galaxy marked as "western source" in Fig. 1. This source is found with a velocity shift of only $(170 \pm 110) \mathrm{km} \mathrm{s}^{-1}$ with respect to the systemic velocity of I Zw 1 and with stellar absorption features of an old stellar population (Canalizo \& Stockton 2001). An ongoing merger with the "western source" is suggested by an extended tidal-tail structure in H I (Lim \& Ho 1999) and by a possible small-scale tidal bridge between the companion and the north-western spiral arm of I Zw 1 (Scharwächter et al. 2003). The bright knot to the north of the disk ("northern source" in Fig. 1) is reported to be a projected foreground star (e.g. Sargent 1970; Stockton 1982; Hutchings \& Crampton 1990). As a Seyfert galaxy, I Zw 1 represents the prototypical NLS1 (Osterbrock \& Pogge 1985) with narrow permitted lines, weak [O III] emission, strong [Fe II] emission, a steep soft Xray spectrum, and with X-ray variability (Sargent 1968; Phillips 1976; Oke \& Lauer 1979; Hutchings \& Crampton 1990; Boller et al. 1996; Véron-Cetty et al. 2004; Gallo et al. 2004). The optical spectrum of I Zw 1 reveals a complex mixture of four different line systems, including a high-excitation narrow-line system ([O III], [Ne III], [N II], [Fe VII], and [S II]) with FWHM $1900 \mathrm{~km} \mathrm{~s}^{-1}$ and blueshifted by $1450 \mathrm{~km} \mathrm{~s}^{-1}$ (Véron-Cetty et al. 2004). Similarly high blueshifts of about $1350 \mathrm{~km} \mathrm{~s}^{-1}$ are exhibited by the NIR coronal lines of [Si VI] $\lambda=19634 \AA$ and [Al IX] $\lambda=20400 \AA$ and might be manifestations of AGNdriven outflows (Schinnerer et al. 1998).

An observational case study of I Zw 1 with focus on the implications of a possible merger-induced star formation and AGN activity is presented in this paper. The data set is based on NIR imaging and spectroscopy made with ISAAC at the Very Large Telescope (VLT) of the European Southern Observatory (ESO) on Cerro Paranal (Chile) and on complementary optical imaging made with EFOSC2 at the $3.6 \mathrm{~m}$ telescope of ESO on La Silla (Chile). The observations and the reduction methods are described in Sect. 2. In Sect. 3.1, the ISAAC images of I Zw 1 are discussed with a focus on morphology. Color images of the I Zw 1 host are presented in Sect. 3.2. The nuclear spectrum of I $\mathrm{Zw} 1$ as well as the spectra of the northern and the western source are analyzed in Sect. 3.3. The results are discussed in Sect. 4 and summarized in Sect. 5 .

\section{Observations and data reduction}

The NIR program for I Zw 1 comprises imaging in the $J$-, $H$-, and $K$ s-bands and long-slit spectroscopy in the $H$ - and $K$ bands. It was carried out with ISAAC at the VLT from July to September $2001^{2}$. The observations relevant for this paper are listed in Table 1. The NIR observations are complemented with standard stars used for the photometric calibration and/or the telluric correction. The optical EFOSC2 images, available in the ESO/ST-ECF Science Archive Facility, are based on observations taken in September 2005.

\subsection{Imaging}

The reduction of the ISAAC images is based on the THELI pipeline (Erben et al. 2005) and cross-checked with independent reductions using IRAF $^{3}$ and the DPUSER software for

\footnotetext{
2 The $J$-band images alone have been used previously for a bulgedisk-decomposition (Scharwächter et al. 2003).

Image Reduction and Analysis Facility written at the National Optical Astronomy Observatories (NOAO) in Tucson, Arizona.
} 
Table 1. Summary of the NIR observations of I Zw 1 discussed in this paper. Column 1: observation mode, Col. 2: target (position angle of the long-slit in the case of the spectroscopic data), Col. 3: filter name and central wavelength $[\mu \mathrm{m}]$, Col. 4: date of observations, Col. 5: detector integration time (DIT), Col. 6: number of exposures pre-averaged on the chip (NDIT), Col. 7: number of exposures stored (NEXP), Col. 8: conditions of air mass during the observations.

\begin{tabular}{|c|c|c|c|c|c|c|c|}
\hline Mode & Target & Filter & Date & DIT [s] & NDIT & NEXP & Air Mass \\
\hline Broad Band & I Zw 1 & $J, 1.250$ & 2001-08-19 & 6.000 & 10 & 5 & $1.263-1.268$ \\
\hline \multirow[t]{8}{*}{ Imaging } & GSPC S234-E & $J, 1.250$ & 2001-08-19 & 3.550 & 3 & 5 & $1.335-1.345$ \\
\hline & GSPC S234-E & $J, 1.250$ & 2001-08-19 & 3.550 & 3 & 5 & $1.394-1.405$ \\
\hline & I Zw 1 & $H, 1.650$ & 2001-08-19 & 6.000 & 10 & 5 & $1.258-1.260$ \\
\hline & GSPC S234-E & $H, 1.650$ & 2001-08-19 & 3.550 & 3 & 5 & $1.346-1.356$ \\
\hline & GSPC S234-E & $H, 1.650$ & 2001-08-19 & 3.550 & 3 & 5 & $1.406-1.418$ \\
\hline & I Zw 1 & $K \mathrm{~s}, 2.160$ & 2001-08-19 & 3.545 & 20 & 5 & $1.258-1.257$ \\
\hline & GSPC S234-E & $K \mathrm{~s}, 2.160$ & 2001-08-19 & 3.550 & 3 & 5 & $1.357-1.368$ \\
\hline & GSPC S234-E & $K \mathrm{~s}, 2.160$ & 2001-08-19 & 3.550 & 3 & 5 & $1.419-1.432$ \\
\hline Low Resolution & I Zw $1\left(0^{\circ}\right)$ & $S K, 2.200$ & 2001-07-02 & 60.000 & 1 & 30 & $1.34-1.28$ \\
\hline \multirow[t]{9}{*}{ Spectroscopy } & HD 166224 & $S K, 2.200$ & 2001-07-02 & 5.000 & 6 & 2 & 1.44 \\
\hline & HD 166224 & $S K, 2.200$ & 2001-07-02 & 5.000 & 6 & 2 & 1.16 \\
\hline & SAO 92129 & $S K, 2.200$ & 2001-07-02 & 40.000 & 1 & 8 & 1.23 \\
\hline & I Zw $1\left(43^{\circ}\right)$ & $S K, 2.200$ & 2001-09-22 & 60.000 & 1 & 30 & $1.33-1.27$ \\
\hline & SAO 92128 & $S K, 2.200$ & 2001-09-22 & 3.550 & 5 & 8 & $1.40-1.38$ \\
\hline & SAO 92129 & $S K, 2.200$ & 2001-09-22 & 10.000 & 4 & 8 & $1.24-1.23$ \\
\hline & I Zw $1\left(43^{\circ}\right)$ & $S H, 1.650$ & 2001-09-22 & 70.000 & 1 & 24 & $1.25-1.27$ \\
\hline & SAO 92128 & $S H, 1.650$ & 2001-09-22 & 3.550 & 5 & 8 & $1.29-1.30$ \\
\hline & SAO 92129 & $S H, 1.650$ & 2001-09-22 & 40.000 & 1 & 8 & $1.23-1.22$ \\
\hline
\end{tabular}

astronomical image analysis ${ }^{4}$. Only the first four frames in each filter contain the object at different positions on the array and are used for the reduction. The fifth frame, which is a pure sky image, is omitted. Before the final co-addition of all images, the THELI reduction includes (i) flat-fielding, (ii) the subtraction of a super-flat, (iii) a correction for the remaining reset anomaly of the array via subtracting the profile of the image collapsed along the $x$-axis, (iv) a bad-pixel identification via weightings of the flat and the individual science frames, and (v) sky-subtraction based on sky models derived from the background in each individual science frame after a suitable object masking. The Gaussian $F W H M$ of the point spread functions in the reduced images yield angular resolutions of about 0.65 , $0 . ' 62$, and $0 . ' 46$ for $J, H$, and $K$ s, respectively.

The night of the imaging observations was classified as photometric at the beginning. The extinction rms, however, became more variable toward the second half of the night when the program for I Zw 1 was executed. GSPC S234-E from the catalog of faint NIR LCO/Palomar NICMOS standards (Persson et al. 1998) was observed twice during the night. Both observation sequences for this standard were taken directly one after the other more than one hour before the I Zw 1 observations. This makes it impossible to crosscheck the stability of the zero-points. Instead, magnitudes from the 2MASS All-Sky Point Source Catalog (IPAC Infrared Science Archive (IRSA), Caltech/JPL) ${ }^{5}$, which are available for a few sources in the ISAAC field-of-view, are used for an alternative cross-check. The zero-points measured in the individual sky-subtracted standard star images one step before co-addition are $\mathrm{ZP}(J)=25.11, \mathrm{ZP}(H)=24.75$, and $\mathrm{ZP}(K \mathrm{~s})=24.24$. These zero-points are corrected for extinction using $0.11 \mathrm{mag}$ (air mass) ${ }^{-1}, 0.06 \mathrm{mag}$ (air mass) $)^{-1}$, and

\footnotetext{
${ }^{4}$ Software package written by Dr. T. Ott, see also Eckart \& Duhoux (1990).

${ }^{5}$ http://irsa.ipac.caltech.edu
}

0.07 mag (air mass) $)^{-1}$ in $J, H$, and $K$ s, respectively, as given on the ISAAC web pages.

The optical EFOSC2 images are reduced via standard reduction steps. The calibration in magnitudes is based on the zeropoints logged on the EFOSC2 web pages. Since the calibration is not completely reliable, the EFOSC2 images are not used for absolute photometry.

The QSO nucleus is saturated in the ISAAC $H$ - and $K$ s-band images as well as in the EFOSC2 images. The nucleus and the central bulge region of the QSO host are, therefore, excluded from the analysis of the imaging data.

\subsection{Spectroscopy}

The spectroscopic data on I Zw 1 were obtained in the $H$ - and the $K$-bands in low-resolution mode $(R \mathrm{~s} \approx 600$ for $H$ and $R \mathrm{~s} \approx 500$ for $K$ ), using a $1^{\prime \prime}$-wide long-slit for settings at position angles of $\mathrm{PA}=0^{\circ}$ and $\mathrm{PA}=+43^{\circ}$ (Fig. 2). The setting at $\mathrm{PA}=+43^{\circ}$ is observed in the $H$ - and $K$-band, the setting at PA $=0^{\circ}$ is only observed in $K$. The $K$-band observations done with the ISAAC $S K$ filter cover a wavelength range of about $7000 \AA$ (18000 $\AA-25000 \AA$ ) and give a dispersion of $7.2 \AA /$ pix. The $F W H M$ of the arc lines is similar to $42 \AA$ which corresponds to $580 \mathrm{~km} \mathrm{~s}^{-1}$. The wavelength range and dispersion of the $H$-band observations done with the ISAAC $S H$ filter are about $4200 \AA$ (14000 $\AA-18200 \AA$ ) and $4.8 \AA /$ pix, respectively, which results in a $F W H M$ of the arc lines of about $27 \AA$ or $500 \mathrm{~km} \mathrm{~s}^{-1}$.

The spectroscopic observations are observed with a nod-onthe-slit technique. The reduction of the two-dimensional raw spectra is done using the ISAAC pipeline recipes and crosschecked with a reduction based on IRAF tasks. The pipeline reduction includes (i) reducing the spectroscopic flat field by computing the normalized difference between frames with lamp on and lamp off, (ii) deriving the slit curvature and wavelength calibration from arc lamp exposures, (iii) tracing the spectral tilt for 


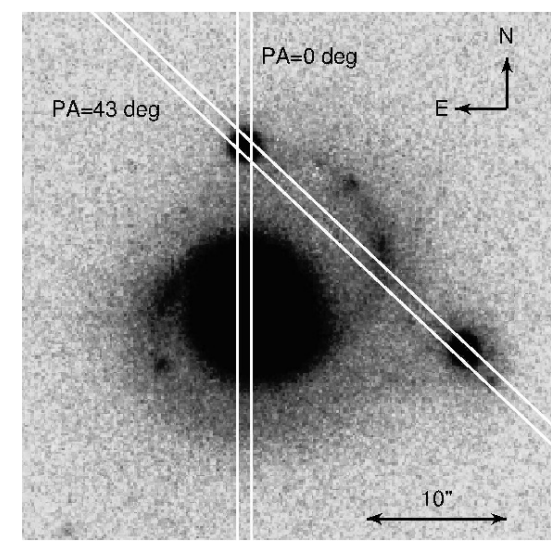

Fig. 2. ISAAC $J$-band image of I Zw 1 overlaid with the two slit settings used for the ISAAC long-slit spectroscopy. The slit for PA $=0^{\circ}$ includes the QSO of I Zw 1 and the northern source, the slit for PA $=43^{\circ}$ includes the northern source and the likely western companion.

different positions along the slit by using exposures of a bright star shifted along the slit, and (iv) the recipe for the final reduction of the science and standard star frames. By this recipe, the frames are flat-fielded and subsets of the observation sequence taken at the same position are identified. The frames of each subset are averaged. These averages are subtracted from each other in both directions so that each difference also has its negative. After the correction for slit curvature and spectral tilt and after wavelength calibration, the subtracted frames are shifted and combined. From the comparison of the wavelength solutions of both reductions using the argon and xenon arc frames and from comparison with the $\mathrm{OH}$ lines, the maximum error in wavelength is estimated to be $0.1 \%$. This accuracy is sufficient for the purpose of this paper.

The contributions of telluric lines and the characteristics of the filter curve present in the object spectra are removed by dividing by a suitable telluric spectrum. The telluric standard stars are reduced exactly like the object spectra. Since all standards are G2V or A0V types, they contain absorption features which are imprinted as emission artifacts on the object spectrum after telluric correction. Most prominent are the higher-series Brackett features in the $H$-band and the $\operatorname{Br} \gamma$ feature in the $K$ band. In the case of the nuclear spectrum of I Zw 1, three standard star observations are available (see Table 1): HD 166224 - a G2V type star with $K$-band magnitude 7.881 (SIMBAD) - observed at two different air masses during the night, and SAO 92129 - a AOV type star with $K$-band magnitude 9.983 (SIMBAD) - observed once during the night. The average spectrum of the two observations of the G2V star HD 166224 better matches the air mass conditions of the object observation and allows a cleaner removal of telluric features (top spectrum in Fig. 7). However, compared to the hydrogen absorptions in the A0V spectrum, the G2V spectrum is characterized by many more absorption features which cause artificial emission lines in the corrected object spectrum. For comparison, both, the spectrum resulting from the telluric correction and calibration with the average spectrum of the two HD 166224 observations and the spectrum resulting from the SAO 92129 calibration, are presented. In the case of the $H$ - and $K$-band spectra for PA $=+43^{\circ}$ (i.e. the spectra of the northern and western source), the spectra of the two available A0V standard star observations are averaged before telluric correction. Since the telluric standard stars frame the object observation in air mass, this average provides a fair approximation of the air mass conditions during the

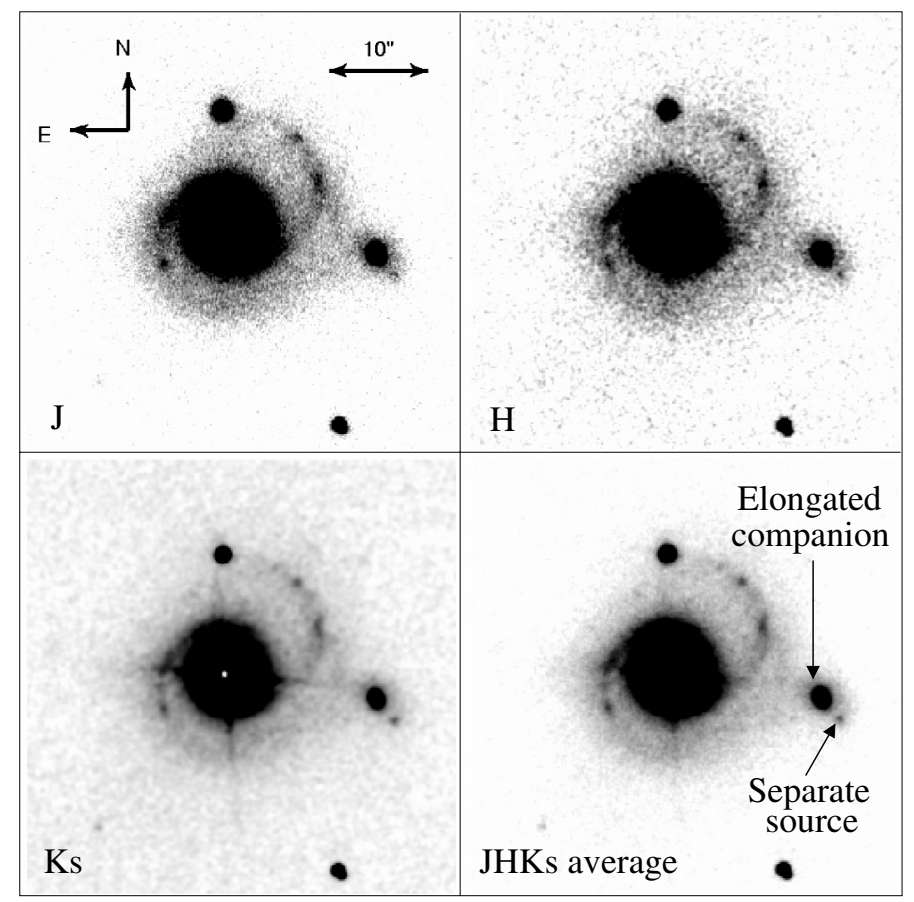

Fig. 3. ISAAC $J_{-}, H$-, and $K$ s-band images of $\mathrm{I} \mathrm{Zw} 1$ as well as an average image of all bands. The latter is computed from the images of all three bands after they have been smoothed to a similar angular resolution, adjusted to a zero background offset, and calibrated in fluxes. All images are shown in inverse colors and with an arbitrary scaling which pronounces the respective features best.

object observations. The resulting object spectrum is compared to a spectrum in which the series of Brackett lines is deblended by a Gaussian fit to the telluric spectrum before telluric division. In order to enhance the signal-to-noise ratio $(\mathrm{S} / \mathrm{N})$ of the spectrum of the northern source, the spectra obtained from the $\mathrm{PA}=+43^{\circ}$ and the $\mathrm{PA}=0^{\circ}$ setting are averaged after correction with the A0V standard stars. Artificial emission lines are clearly marked in the resulting spectra. All spectra are extracted in spatial apertures of $3^{\prime \prime}$ in diameter. Absolute flux calibration is done by multiplying the telluric-corrected object spectra with the black body curves of the telluric standard stars, modeled via the IRAF task STANDARD.

\section{Results}

\subsection{Near-infrared imaging}

The images of I Zw 1 in $J, H$, and $K$ s as well as an image computed from the average of all three bands are shown in Fig. 3. The images provide further evidence of a possible tidal bridge toward the elongated western companion, as already indicated by the $J$-band image alone (Scharwächter et al. 2003). The image in $K \mathrm{~s}$ and the averaged image suggest that the feature to the south-west of the companion resolves into a separate source. This feature was previously interpreted as a kind of tidal tail at the far end of the companion (Scharwächter et al. 2003). The separate source is also resolved in the HST image (Fig. 1). The nature of this source remains unclear. While it might be associated with the likely companion, it could also be a background or foreground object. The object displays a red color in the JHKs color composite in Fig. 5 (see Sect. 3.2). The object is also visible in the spatial cut of the ISAAC spectra belonging to the $\mathrm{PA}=+43^{\circ}$ slit setting and is most pronounced in the $K$-band. 


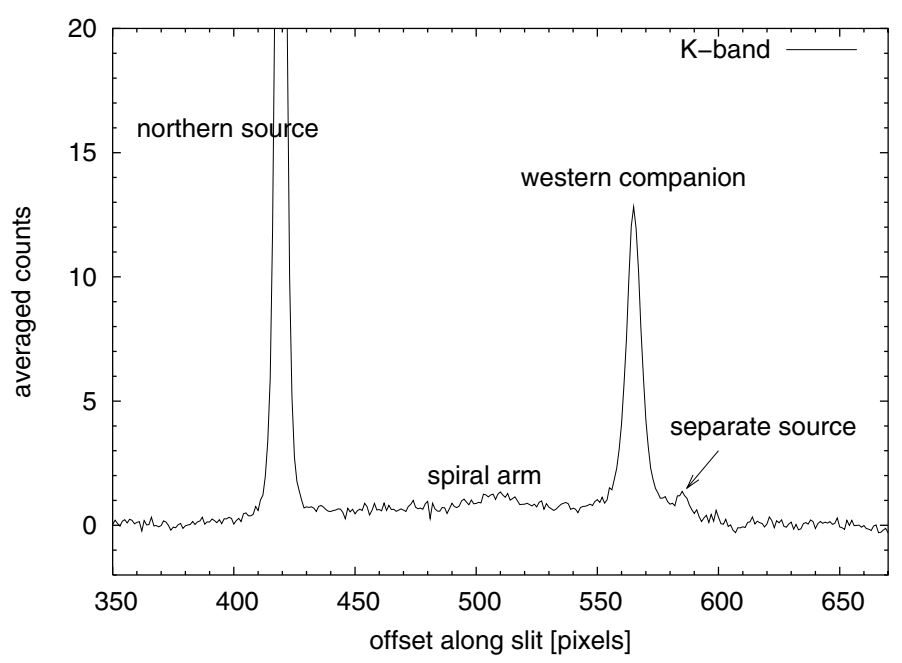

Fig. 4. Cut along the spatial axis of the two-dimensional $K$-band spectrum obtained for the PA $=+43^{\circ}$ setting (see Fig. 2). The cut consists of an average over almost all the spatial lines of the image.

Fig. 4 shows the average of almost all spatial lines of the twodimensional $K$-band spectrum. However, the $\mathrm{S} / \mathrm{N}$ of this separate source is too low for a spectroscopic analysis.

\subsection{Colors}

The optical-to-NIR colors of the I Zw 1 host suggest a concentration of blue stellar populations in the western part of the host galaxy and indicate red colors of the sources to the north and west of the I Zw 1 disk.

The color information is presented in Figs. 5 and 6 in the form of RGB three-color composites and color projections, respectively. The three-color images in Fig. 5 are composed of flux-calibrated images using SAOImage DS9 (see Joye \& Mandel 2003). For each composite, the color distribution of the red, green, and blue input images is uniformly set to a linear scale with upper and lower limits of $95 \%$ about the mean of the pixel distribution. Furthermore, all three images are set to the same bias and contrast values. The $B V R$ and $B V J$ images are shown at the EFOSC2 pixel scale of 0.'314 per pixel and the $B$-band angular resolution of about $1^{\prime \prime}$. The $J$-band image is rebinned and smoothed accordingly. The $J H K$ s image is shown at the ISAAC pixel scale of 0 '. $^{\prime} 1484$ per pixel and the $J$-band angular resolution of 0.65 . A grey-scale representation of the $B-J$ image together with a rough scan of $B-J$ projections across the I Zw 1 host galaxy is presented in Fig. 6. In this figure, all pixels with values lower than $5 \sigma$ of the sky standard deviation in the $B$-band image are neglected. As the scans are intended to mainly highlight east-west color trends, each measurement is averaged over ten pixels in north-south direction. By this method, north-south structures are traced well, while north-south gradients close to the central region are diluted.

The concentration of blue color in the western part of the I Zw 1 host galaxy is most obvious in the $B V J$ composite but is also noticeable in $B V R$ (Fig. 5). In particular, the putative tidal bridge between the north-western spiral arm and the companion galaxy (see Sect. 3.1) is characterized by blue color. The blue color asymmetry between the eastern and the western part of the I Zw 1 host galaxy has not explicitly been noted before. Retrospectively, the effect seems weakly visible in the $B$ - and $I$ band composite presented by Surace et al. (2001, Fig. 2 therein). The much weaker evidence for the color asymmetry in this $B I$

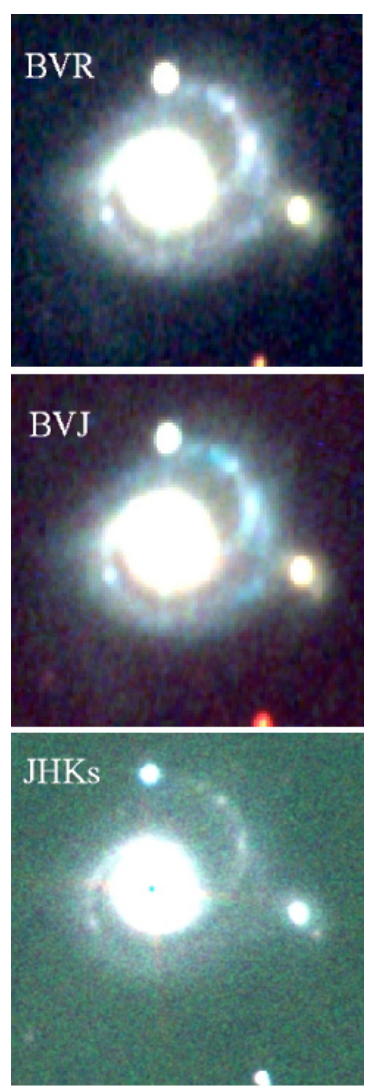

Fig. 5. Optical-to-NIR color composites of I $\mathrm{Zw} 1$. The order of the filters in the labels represents the order of the blue, green, and red channels. The $B V R$ and $B V J$ images are shown at the EFOSC2 pixel scale of 0.314 per pixel and an the $B$-band angular resolution of about $1^{\prime \prime}$. The $J H K$ s image is shown at the ISAAC pixel scale of 0 '. 1484 per pixel and an angular resolution of 0.65 . See text for more details. (The color version of this figure is available in the electronic edition.)

composite compared to Fig. 5 is probably due to the fact that the $B I$ composite is based on a linear interpolation from the $B$ - and $I$ band data and plotted with less contrast on the spiral arm region. A quantitative analysis of the blue color asymmetry in the I Zw 1 host is presented in Fig. 6. The projections in the right panel of this Figure show that there is only one prominent blue region in the eastern part of the host galaxy, i.e. the blue knot seen in projection (2). On the contrary, the western part of the host galaxy is characterized by similarly blue regions in all of the six projections. The blue color suggests an enhanced level of star formation activity. Enhanced star formation activity in this particular part of the I Zw 1 host close to the putative companion galaxy might be causally connected to the supposed minor merger process. It is likely that the star formation is directly induced by the tidal interaction with the western companion galaxy. Enhanced star formation activity in the north-western spiral arm has been discussed in previous publications. Canalizo \& Stockton (2001) conclude that the emission lines in the spectrum of the northwestern spiral arm are typical of H II regions and indicative of ongoing star formation. Similarly, Schinnerer et al. (1998) find star formation activity in the north-western spiral arm by population synthesis for a region about $9^{\prime \prime}$ west and $5^{\prime \prime}$ north of the nucleus of I Zw 1 . They also report this region to be associated with a significant amount of molecular gas.

In the NIR JHKs composite, the I Zw 1 host shows a uniform color. As a NIR composite, this color image mainly traces the old stellar population underlying throughout the host galaxy. 

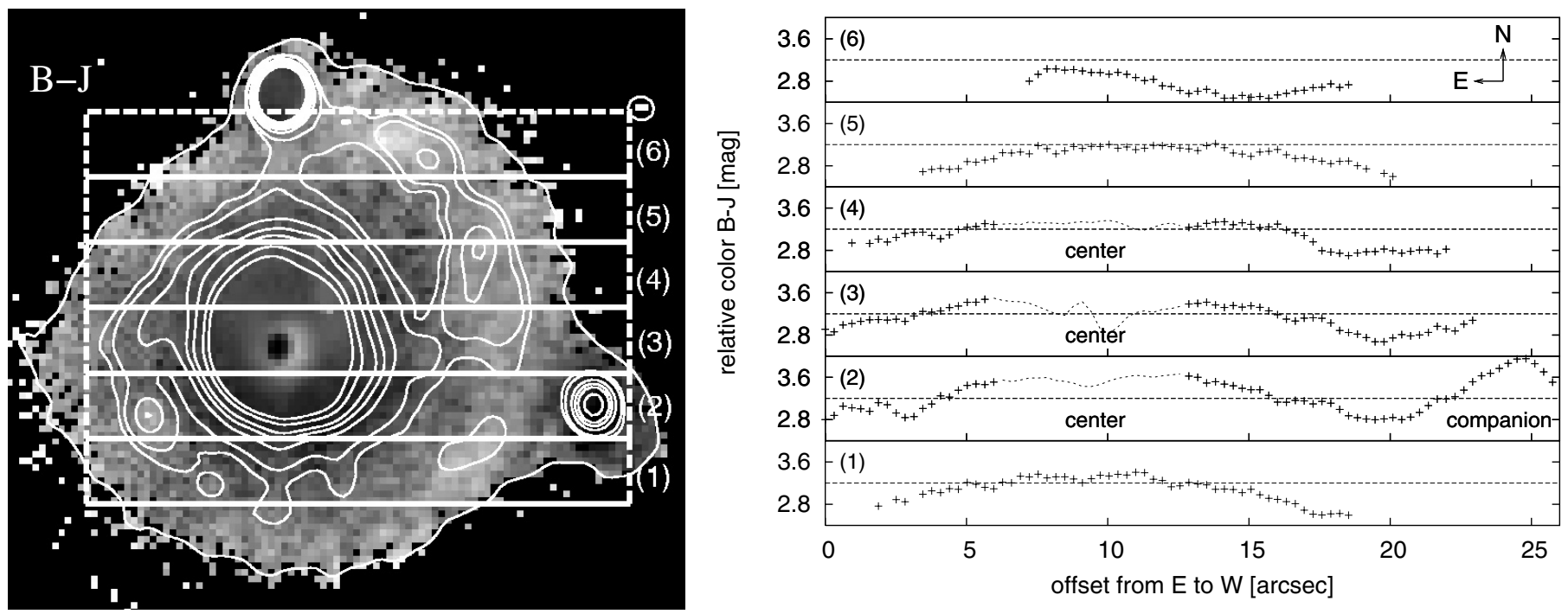

Fig. 6. Relative color trends in $B-J$ for a scan across the host galaxy of I Zw 1 . The left panel shows in grey-scale the $B-J$ image with lighter grey indicating bluer colors. The image is shown at the EFOSC2 pixel scale of 0.314 per pixel and angular resolution of $1^{\prime \prime}$. It is overlaid by white contours from the $B$-band image. The lowest contour shows the $5 \sigma$ level of the standard deviation of the sky in the $B$-band image which is used as a lower limit for pixel rejection (rejected pixels shown in black). For each of the cuts indicated (white boxes) an east-west projection of the mean $B-J$ color is plotted in the graph on the right. The color trends are averaged over ten pixels (i.e. about 3". 1 ) in north-south direction (width of the white boxes). The central region of I $\mathrm{Zw}$ 1, i.e. approximately the region inside the highest contour level in the left panel, is marked by dashed lines in projections (2), (3), and (4). This region is affected by artefacts from image registration and QSO saturation and not reliable for studying color trends. See text for more details.

The analysis of the colors of the QSO nucleus and the central region of the I Zw 1 host is beyond the scope of the data discussed here, as saturation of the QSO nucleus affects the EFOSC2 images and the ISAAC $H$ - and $K s$-band images. Nevertheless, the outskirts of the bulge show indications for a red color in the color images (Fig. 5) and in the $B-J$ projections across the nucleus (Fig. 6). Such a red color might be an indication of an older stellar population. However, the putative circum-nuclear starburst (Schinnerer et al. 1998) and the low mean $J$-band mass-tolight ratio of the bulge (Scharwächter et al. 2003) rather suggest a young stellar population. Alternatively, the reddening could be caused by an increased amount of dust extinction. Dust is suggested by the HST image in Fig. 1 which shows some dust patches in the stellar bulge. Correspondingly, previous NIR studies of I Zw 1 suggest a visual extinction of about 10 mag for the stellar component in the central region of the host galaxy (Eckart et al. 1994; Schinnerer et al. 1998). Finally, as a consequence of the low angular resolution, the red colors might still be dominated by emission from the nucleus. The nuclear colors of I $\mathrm{ZW}_{\mathrm{w}} 1$ as seen in the bands $B, I, H$, and $K^{\prime}$ have previously been reported to show a NIR excess (Surace \& Sanders 1999). According to Figs. 6 and 7 in Surace \& Sanders (1999), this excess can mostly be explained by a hot dust component combined with a QSO spectral energy distribution. Higher angular resolution via adaptive optics or interferometric observations will be needed for a more precise study of color gradients close to the center of I Zw 1.

The northern source and the western source are relatively red objects in each of the color composites in Fig. 5 (see also Fig. 6, in projection (2) for the western source). The red color of the likely northern foreground star agrees with the late stellar type discussed in Sect. 3.3. The red color of the western companion supports the scenario of a predominantly old and evolved stellar population (see Sect. 3.3). The separate object to the south-west of the likely western companion (see Sect. 3.1) appears as a red object in the JHKs composite.

\subsection{Near-infrared spectra}

The nuclear spectrum of I Zw 1 is plotted in Fig. 7. Since the night was not completely photometric, the absolute flux calibration implies uncertainties. The independent calibrations with the two standard stars observed during the same night yield a difference in flux density of about $20 \%$ (Fig. 7). However, within this uncertainty, the mean flux density of the flux-calibrated nuclear spectrum is $2.9 \times 10^{-15} \mathrm{erg} \mathrm{s}^{-1} \mathrm{~cm}^{-2} \AA^{-1}$ or $0.049 \mathrm{Jy}$ and corresponds to a 2 MASS $K$ s magnitude ${ }^{6}$ of 10.4 mag. This agrees with the cataloged 2MASS $K$ s magnitude of I Zw 1 of 10.35 mag (2MASS All-Sky Point-Source Catalog). Previous $K$-band spectra of I Zw 1, measured within similar extraction apertures, are reported to be flux-calibrated by using aperture magnitudes from the literature (Schinnerer et al. 1998; Sosa-Brito et al. 2001). The absolute flux of these spectra is larger than the ISAAC flux by about a factor of 1.5. This corresponds to a magnitude difference of about 0.4. A similar magnitude difference is found in the ISAAC imaging data. The nuclear $J$-band magnitude of I Zw 1 , as derived from the ISAAC image, agrees with the 2MASS measurement but is by about 0.3 to 0.4 mag fainter than a $J$-band measurement taken about nine years earlier (Eckart et al. 1994). While the ISAAC data are saturated in $H$ and $K \mathrm{~s}$, the corresponding measurements from 2MASS show a similar offset with respect to the earlier data by Eckart et al. (1994). If not a calibration offset, this effect might be a manifestation of the NIR variability of I Zw 1 (see e.g. Neugebauer et al. 1989; Neugebauer \& Matthews 1999).

The results from fitting the continuum and the lines in the nuclear ISAAC spectrum of I Zw 1 are shown in Table 2 . The fit is done for the upper spectrum of Fig. 7 by means of the software Specview ${ }^{7}$. The continuum is modeled using a power-law component $f_{\lambda}=f_{\lambda_{\text {ref }}}\left(\lambda / \lambda_{\text {ref }}\right)^{\alpha}$ with $\alpha=0.183, \lambda_{\text {ref }}=21964 \AA$, and

\footnotetext{
6 A $K$-band flux zero-point of $666.8 \mathrm{Jy}$ is adopted (Cohen et al. 2003).

Specview is a product of the Space Telescope Science Institute, which is operated by AURA for NASA.
} 
Table 2. Measured fluxes of the lines identified in the nuclear $K$-band spectrum of I Zw 1 (upper spectrum in Fig. 7). Values in brackets are uncertain measurements only listed for completeness. Column 1: line and rest wavelength, Col. 2: comment about the component of the line, Col. 3: Gaussian FWHM, Col. 4: observed wavelength, Col. 5: flux measured from the ISAAC spectrum, Col. 6: flux published by Schinnerer et al. (1998).

\begin{tabular}{|c|c|c|c|c|c|}
\hline Line & Component & $\begin{array}{l}F W H M^{a} \\
{\left[\mathrm{~km} \mathrm{~s}^{-1}\right]}\end{array}$ & $\begin{array}{l}\lambda_{\mathrm{obs}} \\
{[\AA]}\end{array}$ & $\begin{array}{c}\text { Flux }^{b} \\
{\left[10^{-15} \mathrm{erg} \mathrm{s}^{-1} \mathrm{~cm}^{-2}\right]}\end{array}$ & $\begin{array}{c}\text { Flux (Schinnerer et al. 1998) } \\
{\left[10^{-15} \mathrm{erg} \mathrm{s}^{-1} \mathrm{~cm}^{-2}\right]}\end{array}$ \\
\hline \multicolumn{6}{|c|}{ Permitted lines with narrow and broad components } \\
\hline \multirow[t]{3}{*}{$\operatorname{Pa} \alpha \lambda 18756$} & narrow & 920 & & 86 & \\
\hline & broad & 3110 & & 107 & \\
\hline & total & & 19895 & 193 & $284 \pm 12$ \\
\hline \multirow[t]{3}{*}{$\operatorname{Br} \delta \lambda 19451$} & (narrow & 920 & & 11) & \\
\hline & (broad & 3110 & & 7) & \\
\hline & total & & 20639 & 18 & $3.7 \pm 1.8$ \\
\hline \multirow{3}{*}{$\operatorname{Br} \gamma \lambda 21661$} & (narrow & 920 & & 11) & \\
\hline & (broad & 3110 & & 6) & \\
\hline & total & & 22971 & 17 & $26 \pm 8$ \\
\hline \multicolumn{6}{|c|}{ Narrow forbidden lines with blueshift } \\
\hline [Si VI] $\lambda 19634$ & blue & 920 & 20732 & 7 & $3.5 \pm 1.8$ \\
\hline$\left(+H_{2} S(3) \lambda 19576\right.$ & systemic & & $\sim 20769)$ & & \\
\hline$[\mathrm{A} 1 \mathrm{IX}] \lambda 20400$ & blue & & not detected & & $3.2 \pm 3.2$ \\
\hline$\left(+H_{2} S(2) \lambda 20338\right.$ & systemic & & $\sim 21578)$ & & \\
\hline
\end{tabular}

a The spectral resolution is $650 \mathrm{~km} \mathrm{~s}^{-1}$.

$b$ The $1 \sigma$-error of the flux is $1 \times 10^{-15} \mathrm{erg} \mathrm{s}^{-1} \mathrm{~cm}^{-2}$.

$f_{\lambda_{\text {ref }}}=3.094 \times 10^{-15} \mathrm{erg} \mathrm{s}^{-1} \mathrm{~cm}^{-2} \AA^{-1}$. The hydrogen lines are modeled via the superposition of a broad and a narrow Gaussian component (lower panels in Fig. 7). This two-component model is suggested by the shape of the highest $\mathrm{S} / \mathrm{N}$ hydrogen line $\mathrm{Pa} \alpha$. Only the $\mathrm{Pa} \alpha$ line is used to determine the FWHMs of both Gaussian components. For the fits of the low $\mathrm{S} / \mathrm{N}$ lines $\mathrm{Br} \delta$ and $\operatorname{Br} \gamma$, the $F W H M$ s are taken as fixed parameters. Line centers are determined by eye, and both Gaussian components of each line are constrained to the same center and fitted simultaneously. Out of the two blueshifted high-excitation lines reported by Schinnerer et al. (1998), only [Si VI] is detected on the red wing of the $\operatorname{Br} \delta$ line and modeled with the narrow Gaussian component. [ $\mathrm{Si} \mathrm{VI}$ ] is additionally blended with $H_{2} v=1-0 \mathrm{~S}(3)$ at the systemic velocity of I Zw 1 . This contributes to the uncertainty of the flux measurement for [Si VI] reported in Table 2. As $H_{2} v=1-0 S(1)$ at $\lambda=21218 \AA$ seems to be below the detection limit in Fig. 7 and assuming that the line strength of $H_{2} v=1-0 S(3)$ is less or similar (Kawara et al. 1990), the contribution of $H_{2} v=1-0 S(3)$ to the [Si VI] flux is likely to be small.

The split into two Gaussian components is less certain for the Brackett lines. The corresponding values are parenthesized in Table 2. The fluxes determined for the $\operatorname{Pa} \alpha$ and $\operatorname{Br} \gamma$ lines are by a factor of about 1.5 lower than the fluxes reported by Schinnerer et al. (1998). This is the same factor as found for the disagreement in continuum flux densities. The measured flux of the $\mathrm{Br} \delta$ line shows a different behavior in comparison to the data by Schinnerer et al. (1998). This measurement, however, is the most uncertain one because of the blending with [Si VI] and a possible small contribution from $\mathrm{H}_{2} \mathrm{~S}(3)$. Line ratios (see below) seem to indicate that the new measurement based on the ISAAC data is closer to reality. The rms of the background flux density outside the obvious line emission from I $\mathrm{Zw} 1$ and outside the $\operatorname{Br} \gamma$ artifact is estimated as $3 \times 10^{-17} \mathrm{erg} \mathrm{s}^{-1} \mathrm{~cm}^{-2} \AA^{-1}$. This is a conservative compromise between the less noisy background in the central part of the band and the increasing noise toward the band limits. As an effect of the stronger correction artifacts in the one case and the stronger telluric residuals in the other case, the $\mathrm{G} 2 \mathrm{~V}$ - and the $\mathrm{A} 0 \mathrm{~V}$-corrected spectrum show a similar background rms. Multiplied by the spectral resolution element the given flux density rms corresponds to a $1 \sigma$ flux error of about $1 \times 10^{-15} \mathrm{erg} \mathrm{s}^{-1} \mathrm{~cm}^{-2}$.

The line centers measured for the three hydrogen lines result in a redshift of $z \approx 0.06$. This agrees with the redshift reported for $\mathrm{I} \mathrm{Zw} 1$ in the literature (e.g. Condon et al. 1985). The two $F W H M$ s of the narrow and the broad Gaussian component are similar to the fit of the hydrogen lines in the optical spectrum of I Zw 1 reported by Véron-Cetty et al. (2004). These authors use a narrow Lorentzian component with an $F W H M$ of $1100 \mathrm{~km} \mathrm{~s}^{-1}$ and report the need for an additional broad Gaussian component with an $F W H M$ of $5600 \mathrm{~km} \mathrm{~s}^{-1}$. The putative line center of the [Si VI] feature displays a blueshift of about $1460 \mathrm{~km} \mathrm{~s}^{-1}$ with respect to the velocity of the hydrogen line system of I $\mathrm{Zw} 1$. Schinnerer et al. (1998) report a blueshift of $1350 \mathrm{~km} \mathrm{~s}^{-1}$ for the two high-excitation lines [Si VI] and [Al IX]. Véron-Cetty et al. (2004) speculate that the NIR high-excitation lines may originate from the two high-excitation narrow-line systems, N1 and N2, which they found by fitting the optical spectrum of $\mathrm{I} \mathrm{Zw} 1$. The lines belonging to these systems at optical wavelengths, i.e. [O III], [Ne III], and [N II] (plus [Fe VII] and [S II] in the case of N1), are relatively broad and blueshifted by up to $1450 \mathrm{~km} \mathrm{~s}^{-1}$ in N1. The existence of a blueshifted system of [O III] and [Ne III] lines has also been reported in previous publications (Phillips 1976; Oke \& Lauer 1979; Laor et al. 1997). Similar to other QSOs, the spectrum of I Zw 1 shows a trend of increasing blueshift for lines of increasing ionization level (Laor et al. 1997).

The line ratios computed for the hydrogen lines of Table 2 indicate conditions similar to those in typical $\mathrm{H}$ II regions, possibly affected by a certain amount of dust reddening. This is suggested 

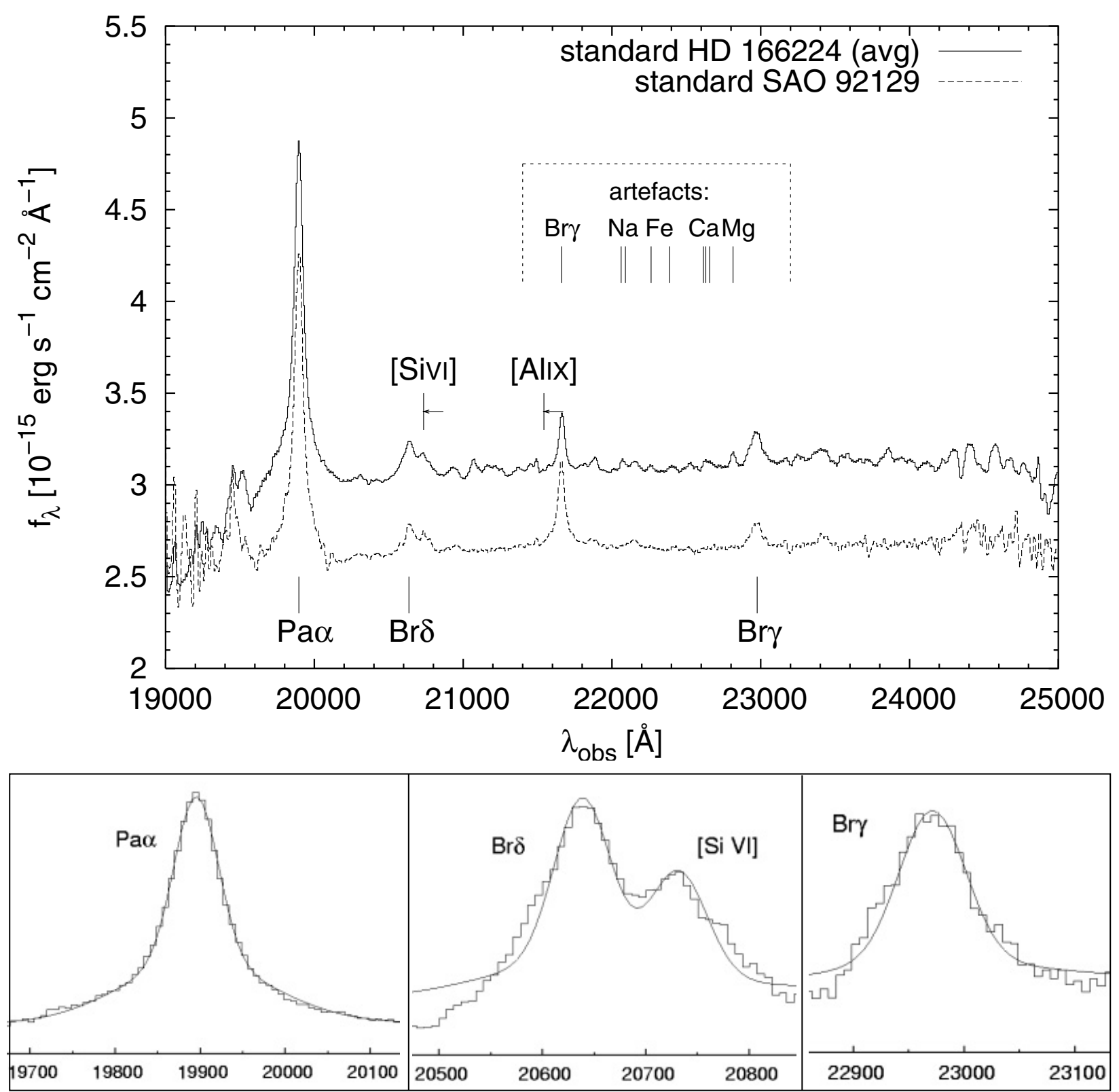

Fig. 7. ISAAC $K$-band spectrum of the nuclear region of I Zw 1 (upper panel) and results for the individual lines from fitting the HD 166224corrected spectrum (lower panels). For a better identification of telluric correction artifacts, the full spectrum is shown as corrected and calibrated by (i) using the average of the two observations of the G2V star HD 166224 and (ii) using the observation of the A0V star SAO 92129. The spectrum is extracted in a $3^{\prime \prime}$-wide aperture centered on the QSO nucleus and boxcar-smoothed to a resolution of about $47 \AA\left(\sim 650 \mathrm{~km} \mathrm{~s}{ }^{-1}\right)$. Prominent lines and telluric-correction artifacts are marked at the respective observed wavelengths. [Si VI] and [Al IX] as well as the arrows visualizing the blueshift are marked according to the positions reported by Schinnerer et al. (1998).

by the fact that the ratios $\operatorname{Pa} \alpha / \operatorname{Br} \gamma \sim 11$ and $\operatorname{Pa} \alpha / \operatorname{Br} \delta \sim 11$ are marginally lower than the theoretical values. Assuming case $\mathrm{B}$ recombination for a hydrogen plasma at a temperature of $10^{4} \mathrm{~K}$ and a density of $10^{4} \mathrm{~cm}^{-3}$ (Hummer \& Storey 1987), the theoretical values result in $\operatorname{Pa} \alpha / \operatorname{Br} \gamma=12.7$ and $\operatorname{Pa} \alpha / \operatorname{Br} \delta=18.34$. These above line ratios are only computed for the total flux of each line because of the uncertainties implied in the twocomponent model for the Brackett lines. For the ratio of $\mathrm{Pa} \alpha$ to $\mathrm{Br} \gamma$ in the nuclear spectrum of I Zw 1, Schinnerer et al. (1998) report a similar value of 11:1. Based on their flux measurements, however, the ratio of $\mathrm{Pa} \alpha$ to $\mathrm{Br} \delta$ results in $\sim 77$. This is unusually high compared to the theoretically expected values. Accordingly, the higher flux of $\operatorname{Br} \delta$ measured in the ISAAC spectrum might be more realistic.
Although less conclusive in consequence of the low $\mathrm{S} / \mathrm{N}$, the $H$ - and $K$-band spectra of the western companion (Fig. 8) seem to support the findings by Canalizo \& Stockton (2001) that the companion shows the spectral properties of an old stellar population. The lines in Fig. 8 are marked at the redshift of $z=0.0616$ derived for the likely companion by Canalizo \& Stockton (2001). Many of these lines match with absorption features in the spectra. In particular, the hydrogen lines are found in absorption rather than emission. This suggests a lack of star formation activity in the western companion.

The $H$ - and $K$-band spectra obtained for the northern source suggest that the source is a cool star at zero redshift and most likely a K-type main-sequence star. This reconfirms the scenario of a foreground star (Sargent 1970; Stockton 1982; 

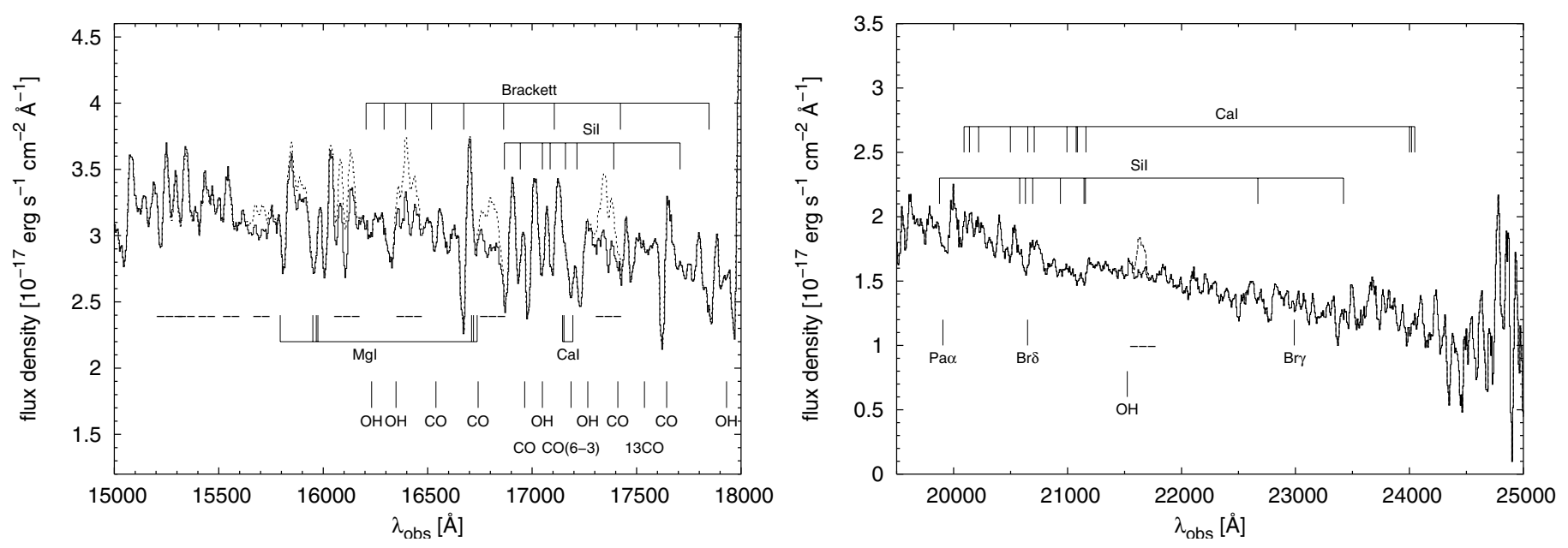

Fig. 8. $H$-band (left panel) and $K$-band (right panel) spectrum of the likely western companion galaxy, both extracted in $3^{\prime \prime}$-wide apertures. The $H$-band spectrum is shown at a resolution of $36 \AA\left(\sim 670 \mathrm{~km} \mathrm{~s}^{-1}\right)$, the $K$-band spectrum at a resolution of $55 \AA\left(\sim 770 \mathrm{~km} \mathrm{~s}{ }^{-1}\right)$. The solid curve shows the spectrum after correction with an A0V telluric spectrum from which the Brackett lines have been deblended. The overlaying thin curve shows the same spectrum corrected without previous deblending. Typical lines in this wavelength region are marked in the plot at a redshift of $z=0.0616$, i.e. the redshift measured for the likely companion by Canalizo \& Stockton (2001). The horizontal dashed markers indicate the wavelength regions contaminated with artificial hydrogen emission in consequence of the telluric correction with A0V standards.

Hutchings \& Crampton 1990), contrary to the alternative scenario of a progenitor nucleus of a galaxy interacting with I Zw 1 or a tidal dwarf galaxy (Davies et al. 2005). The late type of the star is suggested by Fig. 9 which demonstrates that the $H$ and $K$-band spectra of the star agree with the template spectra of local cool stars taken from the library by Lancon \& Rocca-Volmerange (1992). Besides the prominent agreement in $\mathrm{Mg}$ I absorptions, the spectrum of the northern source reflects the continuum shape of cool stars and, in particular, the depression shortward of $16000 \AA$. The latter is attributed to a combined effect of an opacity minimum of $\mathrm{H}^{-}$and water absorption in the atmospheres of cool stars (Lancon \& Rocca-Volmerange 1992).

\section{Discussion}

\subsection{Merger history of I Zw 1}

The consensus from numerical simulations is that major mergers typically result in elliptical remnants (e.g. Hernquist 1992; Naab \& Burkert 2003; González-García \& Balcells 2005; Naab $\&$ Trujillo 2006). The clear spiral structure of I $\mathrm{Zw} 1$ is, therefore, an argument against a recent major merger event. Instead, the evidence for a minor merger between I Zw 1 and the lowmass western companion galaxy is increasing (Lim \& Ho 1999; Canalizo \& Stockton 2001; Scharwächter et al. 2003). The data of the present paper indicate a tidal interaction via the concentration of blue color in the western part of the I Zw 1 host galaxy, i.e. the part which is adjacent to the western source. This leads to the speculation that an ongoing tidal interaction might manifest itself by enhanced star formation activity in those regions of the I Zw 1 host which are facing the western source. Mergerenhanced star formation in the spiral host of $\mathrm{I} Z \mathrm{Zw} 1$ is not unexpected, regarding that the host is suggested to be rich in gas. Based on CO observations, Schinnerer et al. (1998) estimate a total molecular gas mass of about $8 \times 10^{9} \mathcal{M}_{\odot}$ to $10^{10} \mathcal{M}_{\odot}$. Furthermore, enhanced star formation activity has previously been found in the nuclear region and in the north-western spiral arm (Schinnerer et al. 1998; Canalizo \& Stockton 2001).

\subsection{Nature of the western source}

As discussed in Sects. 3.2 and 3.3, the western source does not show any signs for recent star formation. This lack of recent star formation in the western source does not contradict the scenario that the source is in an ongoing merger with I $\mathrm{Zw} 1$. It rather suggests that the companion galaxy does not have a sufficient gas reservoir to incite a starburst, as would be typical for a gaspoor dwarf elliptical. A less plausible alternative is the scenario of a tidal dwarf galaxy. Tidal dwarf galaxies are young dwarf galaxies formed out of the tidal debris of a recent major interaction. They usually have a large gas content and young stellar populations (e.g. Duc et al. 2004). This is in contrast to what is observed for the companion of I Zw 1. Furthermore, tidal dwarfs are typically born along the extended tidal tails formed during a major interaction (e.g. Braine et al. 2001). As discussed before, a recent major interaction between I Zw 1 and a similar-sized galaxy is quite unlikely considering the regular spiral structure of the I Zw 1 host. The above reasoning cannot rule out the possibility that the western companion is an aged tidal dwarf galaxy, i.e. a dwarf galaxy of tidal origin. A tidal origin of dwarf spheroidals around the Milky Way or Andromeda has recently been suggested regarding that dwarf galaxies may appear as dwarf spheroidals after a Hubble time of evolution in the dark matter halo of the primary galaxy (Metz \& Kroupa 2007).

\subsection{Nuclear activity of I Zw 1}

As an infrared-excess Palomar Green QSO, I Zw 1 has been discussed as a possible QSO at a transition stage in the putative evolutionary sequence from ULIRGs to QSOs (e.g. Sanders et al. 1988; Surace et al. 2001; Canalizo \& Stockton 2001). According to this scenario the QSO activity in I Zw 1 would be young. A young stage of the AGN in I Zw 1 is also suggested via a different line of argument: similarities between NLS1s and broad-absorption line QSOs gave rise to the hypothesis that at least those NLS1s with outflows could be counterparts of high-redshift, high-luminosity broad-absorption line QSOs (e.g. Leighly et al. 1997; Brandt \& Gallagher 2000; Mathur 2000). As one alternative, both classes of objects could be observed during 

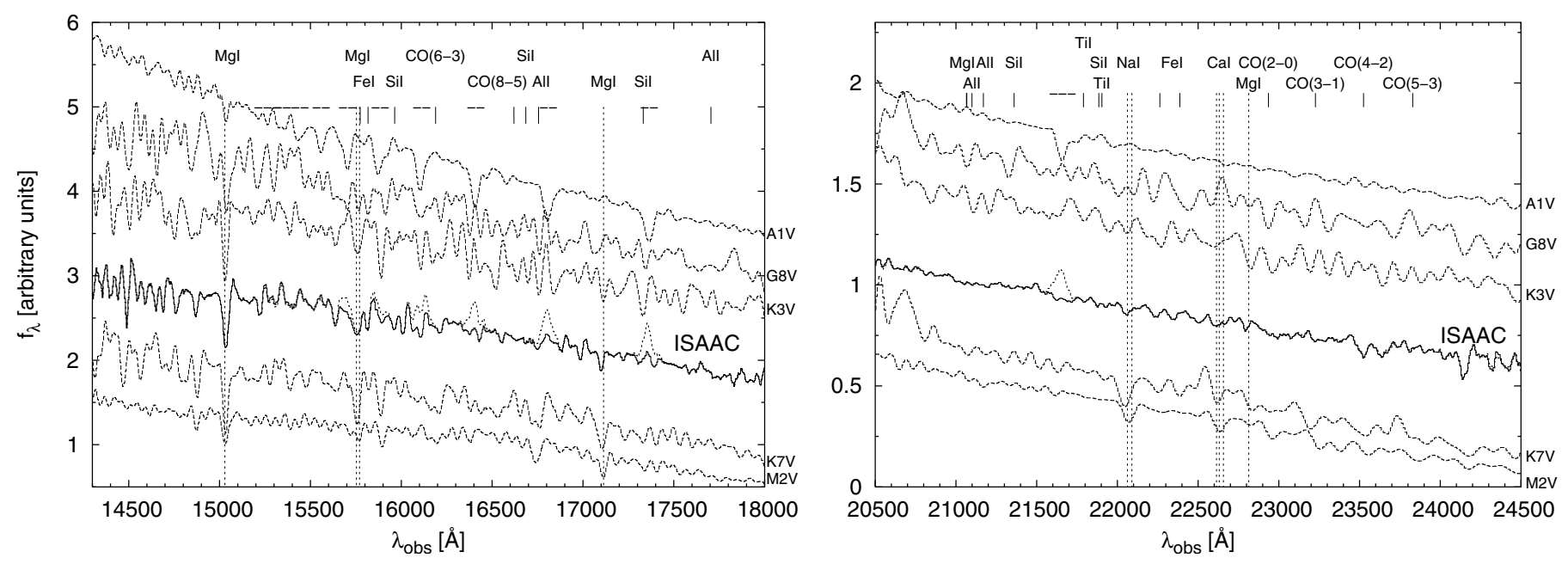

Fig. 9. $H$-band (left panel) and $K$-band (right panel) spectrum of the northern source, both extracted in $3^{\prime \prime}$-wide apertures and compared to stellar templates (see labels) from the library by Lancon \& Rocca-Volmerange (1992). The ISAAC spectrum (solid curve) is derived from the telluric correction with an A0V spectrum with deplended Brackett lines. It is overlaid with the spectrum corrected without deblending (thin curve). The spectral resolutions are $36 \AA\left(\sim 670 \mathrm{~km} \mathrm{~s}^{-1}\right)$ and $55 \AA\left(\sim 770 \mathrm{~km} \mathrm{~s}^{-1}\right)$ in $H$ and $K$, respectively. The library spectra have similar spectral resolutions. For the purpose of comparison with the ISAAC spectra, the available units of wavenumber (in $\mathrm{cm}^{-1}$ ) versus arbitrary units of flux per unit frequency are converted into wavelength (in $\AA$ ) versus flux per unit wavelength. All spectra are normalized to a flux of 1 at $21500 \AA$ and shifted along the flux-axis for this representation. Typical lines in this wavelength region are labeled. The lines identified in the ISAAC spectra are marked by a vertical dashed line. The horizontal dashed lines indicate the Balmer lines which artificially appear as emission lines in the ISAAC spectra as a consequence of the telluric correction via A0V stars.

a young phase of AGN evolution, characterized by smaller black hole masses and higher accretion rates (e.g. Pounds et al. 1995; Mathur 2000; Yuan \& Wills 2003; Maiolino et al. 2004; Botte et al. 2004). In fact, I Zw 1 is a NLS1 whose similarity with broad-absorption line QSOs has previously been noted. The strong optical Fe II emission of I Zw 1, the weak [O III] emission, the "red" UV continuum, and the strong infrared emission remind of low-ionization broad-absorption line QSOs (Laor et al. 1997). Furthermore, the blueshifts of high-excitation lines in the optical and NIR spectra, as reported by Schinnerer et al. (1998), Véron-Cetty et al. (2004), and in the present paper, suggest an AGN-driven outflow. Although these arguments suggest a young stage of the AGN in I Zw 1, it cannot be excluded that the AGN might just appear young.

Furthermore, it remains unclear which process is responsible for the nuclear activity in I Zw 1 and which part is assigned to the tidal interaction with the western companion galaxy. The conclusions from minor-merger simulations by Hernquist \& Mihos (1995) suggest that the companion galaxy of I Zw 1 might still be too distant to be the cause of the AGN in I Zw 1 . The simulations rather indicate that a minor merger may induce central activity in the primary galaxy during the late stages of the merger, i.e. when the satellite galaxy has reached the central few kiloparsecs of the primary galaxy (Hernquist \& Mihos 1995). Disk star formation, on the contrary, may well be induced during the early stages of the merger. Thus, the disk star formation indicated by the blue colors in the western part of the I Zw 1 host galaxy might indeed be directly connected to the interaction with the western companion. The role of tidal interactions in the formation of AGN in Seyfert galaxies is generally controversial (see review by Schmitt 2004). Corbin (2000) suggests that instead of triggering nuclear activity minor mergers might rather increase the existing AGN luminosity to QSO levels. It is possible that the previously existing Seyfert nucleus of I Zw 1 is raised to QSO luminosities by the increased AGN fueling rate due to the minor merger with the western companion galaxy. The onset of the nuclear activity in I Zw 1 might instead be related to a mergerindependent AGN triggering mechanism. A possible mechanism for merger-independent triggering of Seyfert activity could be the stochastic accretion model shown by Hopkins \& Hernquist (2006). As discussed above, the AGN triggering by a previous strong merger event in the case of I Zw 1 is unlikely because of the clear spiral host galaxy.

\section{Summary}

1. An ongoing tidal interaction between I Zw 1 and the western source is likely to be responsible for enhanced star formation activity in the western part of the I Zw 1 host galaxy. This is suggested by the concentration of blue color in the part of the I $\mathrm{Zw} 1$ host galaxy which is adjacent to the western companion galaxy.

2. In agreement with previous studies, the NIR spectra and the optical-to-NIR color images of the western companion galaxy indicate an old evolved stellar population without recent star formation. This suggests that the western companion galaxy is a gas-poor dwarf elliptical. An extension to the west of the likely companion resolves into a separate object.

3 . The NIR spectra of the northern source reconfirm the scenario of a late-type foreground star, as reported by Sargent (1970) and Stockton (1982).

4. In agreement with Schinnerer et al. (1998), the hydrogen line ratios indicate no significant amount of reddening toward the very nucleus of I Zw 1 . Out of the two blueshifted highexcitation lines reported by Schinnerer et al. (1998) only [Si VI] is marginally detected.

5. The outskirts of the bulge region of I Zw 1 have redder colors than the disk. This might be an indication for a predominance of old stars and/or an increased amount of dust in this region. At the low angular resolution, the color might alternatively be dominated by the NIR excess from the nucleus (Surace \& Sanders 1999). Higher angular resolution is 
needed for verifying the previous signs for young stellar populations in the central region of the bulge (Schinnerer et al. 1998; Scharwächter et al. 2003).

Acknowledgements. We would like to thank the anonymous referee for a helpful report. We are grateful to the anonymous observer who carried out the VLT observations in service mode. J.S. thanks Dr. M. Schirmer for his advice with the installation and use of the THELI pipeline. Parts of the research were supported by the Deutsche Forschungsgemeinschaft (DFG) via grant SFB 494 and by the "Studienstiftung des deutschen Volkes" via a scholarship for doctoral students for J.S. until June 2004.

\section{References}

Boller, T., Brandt, W. N., \& Fink, H. 1996, A\&A, 305, 53

Botte, V., Ciroi, S., Rafanelli, P., \& Di Mille, F. 2004, AJ, 127, 3168

Braine, J., Duc, P.-A., Lisenfeld, U., et al. 2001, A\&A, 378, 51

Brandt, W. N., \& Gallagher, S. C. 2000, New Astron. Rev., 44, 461

Bushouse, H. A., Borne, K. D., Colina, L., et al. 2002, ApJS, 138, 1

Canalizo, G., \& Stockton, A. 2001, ApJ, 555, 719

Cohen, M., Wheaton, W. A., \& Megeath, S. T. 2003, AJ, 126, 1090

Condon, J. J., Hutchings, J. B., \& Gower, A. C. 1985, AJ, 90, 1642

Corbin, M. R. 2000, ApJ, 536, L73

Dahari, O. 1984, AJ, 89, 966

Dasyra, K. M., Tacconi, L. J., Davies, R. I., et al. 2007, ApJ, 657, 102

Davies, R., Tacconi, L., Genzel, R., \& Thatte, N. 2005, in Science with Adaptive Optics, ed. W. Brandner \& M. Kasper, ESO Astrophysics Symposia de Robertis, M. M., Yee, H. K. C., \& Hayhoe, K. 1998, ApJ, 496, 93

Di Matteo, T., Springel, V., \& Hernquist, L. 2005, Nature, 433, 604

Duc, P.-A., Bournaud, F., \& Masset, F. 2004, A\&A, 427, 803

Dultzin-Hacyan, D., Krongold, Y., Fuentes-Guridi, I., \& Marziani, P. 1999, ApJ, 513, L111

Dunlop, J. S., McLure, R. J., Kukula, M. J., et al. 2003, MNRAS, 340, 1095

Eckart, A., \& Duhoux, P. R. M. 1990, in Astrophysics with Infrared Arrays, ed. R. Elston, ASP Conf. Ser. 14, 336

Eckart, A., van der Werf, P. P., Hofmann, R., \& Harris, A. I. 1994, ApJ, 424, 627

Erben, T., Schirmer, M., Dietrich, J. P., et al. 2005, Astron. Nachr., 326, 432

Gallo, L. C., Boller, T., Brandt, W. N., Fabian, A. C., \& Vaughan, S. 2004, A\&A, 417,29

González-García, A. C., \& Balcells, M. 2005, MNRAS, 357, 753

Grogin, N. A., Conselice, C. J., Chatzichristou, E., et al. 2005, ApJ, 627, L97

Haas, M., Klaas, U., Müller, S. A. H., et al. 2003, A\&A, 402, 87

Hernquist, L. 1992, ApJ, 400, 460

Hernquist, L., \& Mihos, J. C. 1995, ApJ, 448, 41

Hopkins, P. F., \& Hernquist, L. 2006, ApJS, 166, 1

Hopkins, P. F., Hernquist, L., Cox, T. J., et al. 2005, ApJ, 630, 705

Hopkins, P. F., Hernquist, L., Cox, T. J., et al. 2006, ApJS, 163, 1

Hummer, D. G., \& Storey, P. J. 1987, MNRAS, 224, 801
Hutchings, J. B., \& Crampton, D. 1990, AJ, 99, 37

Joye, W. A., \& Mandel, E. 2003, in Astronomical Data Analysis Software and Systems XII, ed. H. E. Payne, R. I. Jedrzejewski, \& R. N. Hook, ASP Conf. Ser. 295, 489

Kauffmann, G., Heckman, T. M., Tremonti, C., et al. 2003, MNRAS, 346, 1055 Kawara, K., Nishida, M., \& Gregory, B. 1990, ApJ, 352, 433

Koulouridis, E., Chavushyan, V., Plionis, M., Krongold, Y., \& Dultzin-Hacyan, D. 2006, ApJ, 651, 93

Lancon, A., \& Rocca-Volmerange, B. 1992, A\&AS, 96, 593

Laor, A., Jannuzi, B. T., Green, R. F., \& Boroson, T. A. 1997, ApJ, 489, 656

Laurikainen, E., \& Salo, H. 1995, A\&A, 293, 683

Leighly, K. M., Mushotzky, R. F., Nandra, K., \& Forster, K. 1997, ApJ, 489, L25

Lim, J., \& Ho, P. T. P. 1999, ApJ, 510, L7

Maiolino, R., Oliva, E., Ghinassi, F., et al. 2004, A\&A, 420, 889

Mathur, S. 2000, MNRAS, 314, L17

Metz, M., \& Kroupa, P. 2007, ArXiv Astrophysics e-prints

Naab, T., \& Burkert, A. 2003, ApJ, 597, 893

Naab, T., \& Trujillo, I. 2006, MNRAS, 369, 625

Neugebauer, G., \& Matthews, K. 1999, AJ, 118, 35

Neugebauer, G., Soifer, B. T., Matthews, K., \& Elias, J. H. 1989, AJ, 97, 957

Oke, J. B., \& Lauer, T. R. 1979, ApJ, 230, 360

Osterbrock, D. E., \& Pogge, R. W. 1985, ApJ, 297, 166

Persson, S. E., Murphy, D. C., Krzeminski, W., Roth, M., \& Rieke, M. J. 1998, AJ, 116, 2475

Phillips, M. M. 1976, ApJ, 208, 37

Pounds, K. A., Done, C., \& Osborne, J. P. 1995, MNRAS, 277, L5

Rafanelli, P., Violato, M., \& Baruffolo, A. 1995, AJ, 109, 1546

Sanders, D. B., Soifer, B. T., Elias, J. H., et al. 1988, ApJ, 325, 74

Sargent, W. L. W. 1968, ApJ, 152, L31

Sargent, W. L. W. 1970, ApJ, 160, 405

Scharwächter, J., Eckart, A., Pfalzner, S., et al. 2003, A\&A, 405, 959

Schinnerer, E., Eckart, A., \& Tacconi, L. J. 1998, ApJ, 500, 147

Schmidt, M., \& Green, R. F. 1983, ApJ, 269, 352

Schmitt, H. R. 2004, in IAU Symposium, ed. T. Storchi-Bergmann, L. C. Ho, \& H. R. Schmitt, 395

Sosa-Brito, R. M., Tacconi-Garman, L. E., Lehnert, M. D., \& Gallimore, J. F. 2001, ApJS, 136, 61

Staguhn, J. G., Schinnerer, E., Eckart, A., \& Scharwächter, J. 2004, ApJ, 609, 85

Stockton, A. 1982, ApJ, 257, 33

Surace, J. A., \& Sanders, D. B. 1999, ApJ, 512, 162

Surace, J. A., Sanders, D. B., \& Evans, A. S. 2001, AJ, 122, 2791

Tacconi, L. J., Genzel, R., Lutz, D., et al. 2002, ApJ, 580, 73

Urry, C. 2004, in AGN Physics with the Sloan Digital Sky Survey, ed. G. T. Richards \& P. B. Hall, ASP Conf. Ser. 311, 49

Veilleux, S., Kim, D.-C., \& Sanders, D. B. 2002, ApJS, 143, 315

Véron-Cetty, M.-P., Joly, M., \& Véron, P. 2004, A\&A, 417, 515

Yuan, M. J., \& Wills, B. J. 2003, ApJ, 593, L11 